\title{
Surface Adjustment Strategy for a Large Radio Telescope with Adjustable Dual Reflectors
}

\author{
Peiyuan Lian ${ }^{1}$, Congsi Wang ${ }^{1 *}$, Song Xue ${ }^{1}$, Qian $\mathrm{Xu}^{2}, \mathrm{Yu} \mathrm{Shi}^{3}, \mathrm{Yu} \mathrm{Jia}^{3}$, Binbin Xiang ${ }^{2}$, Yan Wang ${ }^{1}$, \\ Yuefei Yan ${ }^{1}$ \\ ${ }^{1}$ School of Electromechanical Engineering, Xidian University, No.2 Taibai South Road, Xi'an 710071, China. \\ ${ }^{2}$ Xinjiang Astronomical Observation, Chinese Academy of Sciences, No.150 Science 1-Street, Urumqi 830011, \\ China \\ ${ }^{3}$ Department of Mechanical Engineering, University of Chester, Chester, CH2 4NU, UK \\ *ongsiwang@163.com
}

\begin{abstract}
With the development of large-aperture and high-frequency radio telescopes, a surface adjustment procedure for the compensation of surface deformations has become of great importance. In this paper, an innovative surface adjustment strategy is proposed to achieve an automated adjustment for the large radio telescope with adjustable dual reflectors. In the proposed strategy, a high-precision and long-distance measurement instrument is adopted and installed on the back of the sub-reflector to measure the distances and elevation angles of the target points on the main reflector. Here, two surface adjustment purposes are discussed. The first purpose is to ensure that the main reflector and the sub-reflector are always positioned at their ideal locations during operation. The second purpose is to adjust the main reflector to the location of the best fitting reflector, and the sub-reflector to the focus of the best fitting reflector. Next, the calculation procedures for the adjustments of the main reflector and the sub-reflector are discussed in detail, and corresponding simulations are carried out to verify the proposed method. The results show that the proposed strategy is effective. This paper can provide helpful guidance for the design of automated surface adjustments for large telescopes.
\end{abstract}

\section{Introduction}

Large radio telescopes have been widely used in deep space exploration and radio astronomy because of their high gain and narrow beams [1]. The common features of this kind of telescope are a large aperture and a high operating frequency. A high operating frequency requires a high surface accuracy, especially for millimetre and sub-millimetre telescopes, while a large aperture can reduce the surface accuracy. More specifically, a large aperture can result in a large gravitational deformation, and the telescope can also become more sensitive to inevitable external environmental impacts, such as temperature and wind [2-4]. Numerous research papers have analysed the effect of the surface deformation of a radio telescope on its electromagnetic performance [5-8].

To reduce the surface deformation, the traditional method is to increase the structure stiffness as much as possible and achieve an acceptable precision, which can be impractical both from economical and technological points of view. A homologous design was first proposed by S. von Hoerner to overcome the gravitational limit and has been further studied by many other researchers [9-11]. A homologous design allows the reflector surface to deform from one paraboloid to another while achieving a reduction in weight. To further improve the telescope's electromagnetic performance, an integrated structural-electromagnetic design method has been proposed and studied by many researchers, which can directly select the electromagnetic performance as the optimization object, and good results have been achieved [12-15]. However, with the trend towards larger apertures as well as higher operating frequencies, it has become impossible for these traditional methods to meet all of the stringent accuracy requirements.
For a modern radio telescope, an active main reflector has been considered to be the best choice for meeting the stringent accuracy requirement. Each panel of the active main reflector is fixed to four actuators at its four corners. Compared with the traditional telescopes, the screws are replaced by the actuators to uphold the panels, which will greatly improve the panel's adjustment capability.

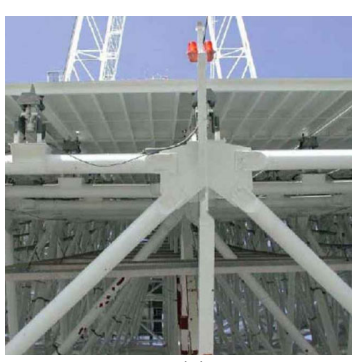

(a)

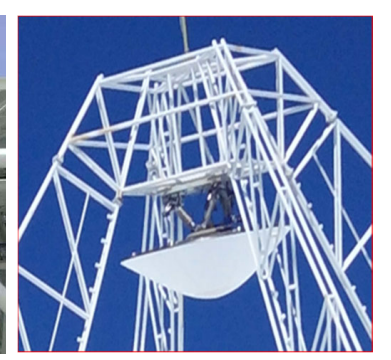

(b)
Fig. 1 Adjustable structures

(a) An adjustable main reflector; (b) An adjustable subreflector

Fig. 1 shows images of an active main reflector and an adjustable sub-reflector. Taking the Tianma (TM) telescope with an aperture of $50 \mathrm{~m}$ located in Shanghai as an example [4], the sub-reflector is installed on a Stewart platform to perform the adjustment, and the main reflector can be directly adjusted by 1104 actuators with a surface root mean square (rms) accuracy of $0.3 \mathrm{~mm}$. The $50 \mathrm{~m}$ Large Millimeter Telescope (LMT) at the summit of Sierra Negra and the $50 \mathrm{~m}$ Large Sub-millimeter Telescope (LST) planned to be built have higher frequencies [16-17], and the $100 \mathrm{~m}$ Green Bank Telescope (GBT) in West Virginia and the $110 \mathrm{~m}$ Qitai 
Telescope (QTT) planned to be built have larger apertures [18-19]. The active main reflector can easily compensate for the gravitational deformation using the table search method, and the surface accuracy can be greatly improved. However, for deformations caused by time-varying temperature and wind, it will be very difficult to realize compensation because the deformations are unknown. Many researchers have studied the compensation methods for the effects of wind and temperature [20-21]. For example, pressure sensors can be adopted in studies related to wind, and a thermal control system with a closed-up backup structure can be used to minimize the effect of temperature. Ultimately, this approach incurs a high cost, and it is still very difficult to achieve satisfactory results in engineering applications.

If the main reflector deformation and sub-reflector shift could be automatically determined in real time, all the above problems would be simplified. Therefore, in this field, measurement technology has become a research hotspot. There are two methods commonly used for deformation measurement. The first method involves directly measuring the spatial coordinates of the reflector's nodes, which can be achieved with a laser measurement system or a photogrammetry system. In engineering, a laser measurement system is generally used in the panel installation process on the ground, and the process can be very time-consuming because all of these single points have to be measured manually. A photogrammetry system can be easily adopted in the measurement process of the whole reflector after all panels are installed. However, a manual measurement will result in difficulties in the real-time measurement of timevarying deformations. In addition, the main reflector and subreflector must match each other to a high performance, so the perfect compensation of the sub-reflector shift is also very important [22]. Unfortunately, determining the sub-reflector shift and amount of adjustment is also not easy. The common practice in engineering is to measure the telescope's far-field power pattern and then adjust the sub-reflector iteratively based on the levels of the side lobes and gain. Obviously, the whole adjustment process is ineffective and cannot be achieved automatically. Another method is holography [2324], which has been successfully applied to some large telescopes. The out-of-focus (OOF) technique is a typical method that is used to estimate the main reflector's largescale errors. This technique requires several out-of-focus images of a compact source and the signal-to-noise ratio needs to be very good. This means that the measurement process cannot be carried out during the working period or for some attitudes. Thus, holography is also not suitable for automation.

In this paper, a laser measurement system is adopted because of its high precision and long measurement distance. At the same time, considering that the sub-reflector must match with the main reflector, to avoid the measurement procedure of the sub-reflector, we install the measurement system on the back of the sub-reflector. In this case, the measurement data of the main reflector are in the coordinate system attached to the sub-reflector, and the relationship between the main reflector and the sub-reflector can be easily established. In fact, for a large telescope, the size of the subreflector is large enough for the installation of the measurement system. Here, we assume that this can be realized through the design of a suitable mechanism, such as designing the wheel rail to be along the edge of the sub- reflector or the round door hole to be in the centre of the subreflector. The laser tracker produced by the companies API or Faro can be taken as an example because of its micronlevel measurement accuracy, measurement distance of dozens of metres, and automatic target search ability. Based on these above assumptions, two surface adjustment procedures will be discussed. The first procedure ensures that the main reflector and the sub-reflector are always positioned at their ideal locations during telescope operation, and the second procedure adjusts the main reflector to the location of the best fitting reflector and the sub-reflector to the focus of the best fitting reflector. These two adjustment strategies will be discussed in detail, and corresponding simulations will be presented in the following sections.

\section{Pattern analysis of dual reflectors}

Fig. 2 shows a geometric diagram of the Cassegrain dual-reflector telescope, where $a$ represents half the length of the short axis and $c$ represents half the length of the focal length of the sub-reflector and $\theta_{f}$ and $\theta_{p}$ represent the two angles shown in Fig. 2.

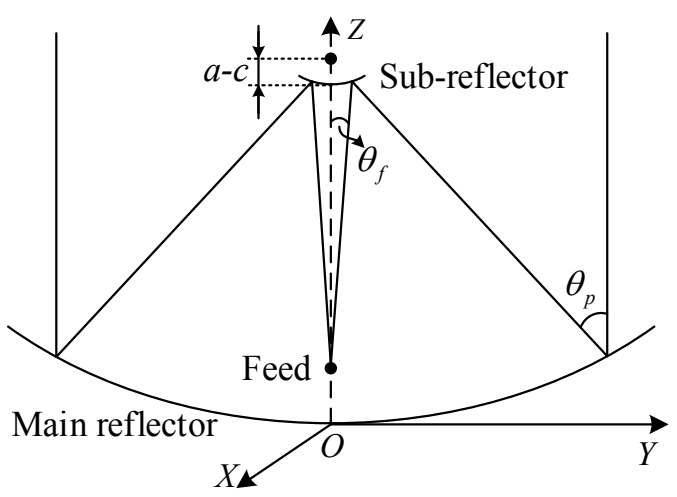

Fig. 2 Geometric diagram of the Cassegrain dual-reflector antenna

Assume that the point $(x, y, z)$ on the surface of the main reflector will undergo a displacement $\left(\Delta x_{p}, \Delta y_{p}, \Delta z_{p}\right)$. The resulting change in the optical path length can be presented as [25-26]

$$
\delta_{p}=2 \cdot\left(\Delta x_{p} \cos \alpha+\Delta y_{p} \cos \beta+\Delta z_{p} \cos \gamma\right) \cdot \cos \gamma
$$

where $\cos \alpha, \cos \beta$ and $\cos \gamma$ are the direction cosines of the unit normal vector, $\cos \alpha=\cos \phi, \cos \beta=\sin \phi$, $\cos \gamma=\cos \left(\theta_{p} / 2\right), \phi=\arctan (y / x), \theta_{p}=2 \arctan (r /(2 f))$, $\theta_{f}=2 \arctan (r /(2 M f)), r=\sqrt{x^{2}+y^{2}}$, and $f$ and $M f$ are the focal lengths of the main reflector and the equivalent prime focus paraboloidal reflector, respectively.

Assuming that the sub-reflector's rigid-body translation and rotation are $\left(\Delta x_{s}, \Delta y_{s}, \Delta z_{s}\right)$ and $\left(\psi_{x s}, \psi_{y s}\right)$, respectively, the resultant expression for the change in the optical path length can be written as follows [25-26]

$$
\begin{gathered}
\delta_{s}=\left(-\Delta x_{s} \cos \phi-\Delta y_{s} \sin \phi\right)\left(\sin \theta_{p}-\sin \theta_{f}\right)+ \\
\Delta z_{s}\left(\cos \theta_{p}+\cos \theta_{f}\right)+(c-a) . \\
\left(\psi_{x s} \sin \phi-\psi_{y s} \cos \phi\right) \cdot\left(\sin \theta_{p}+M \sin \theta_{f}\right)
\end{gathered}
$$


where $\sin \theta_{f}=\frac{r /(M f)}{1+(r /(2 M f))^{2}}, \sin \theta_{p}=\frac{r / f}{1+(r / 2 f)^{2}}$, and for the Gregorian dual-reflector telescope, $\left(\sin \theta_{p}-\sin \theta_{f}\right)$ in equation (2) should be replaced by $\left(\sin \theta_{p}+\sin \theta_{f}\right)$.

Because the relationship between the aperture field and the far field is a Fourier transform pair, with the additional effects of $\delta_{p}$ and $\delta_{s}$, the far field pattern can be calculated by the following formula

$$
E(\tilde{\theta}, \tilde{\phi})=\iint_{A} F(r, \phi) e^{j k r \sin \tilde{\theta} \cos (\tilde{\phi}-\phi)} e^{j k\left(\delta_{p}+\delta_{s}\right)} r d r d \phi
$$

where $A$ is the projected area of the reflector on the $O-X Y$ plane, $(\tilde{\theta}, \tilde{\phi})$ is the observation direction, $F(r, \phi)$ is the aperture amplitude distribution, $k=2 \pi / \lambda$, and $\lambda$ is the wave length.

\section{Measurement strategy}

Fig. 3 shows a diagram of an ideal dual-reflector telescope and laser measurement system. Here, we refer to $O_{r}-x_{r} y_{r} z_{r}$ as the reference coordinate system, where $O_{r}$ is the origin of the main reflector and $O_{r}-z_{r}$ coincides with the main reflector's axis. For the active main reflector, the panels are fixed to the actuators, and for the traditional main reflector, the panels are fixed to screws. Then the measurement targets are pasted on the main reflector near the actuators for the active main reflector or near the vertices of the panels for the traditional main reflector, and the ideal coordinates of the measurement targets in $O_{r}-x_{r} y_{r} z_{r}$ are denoted by $\left(x_{a}^{r}, y_{a}^{r}, z_{a}^{r}\right)$, where $a=1,2, \cdots, N$ and $N$ is the total number of targets. The measurement instrument is installed on the back of the subreflector and the instrument coordinate system is denoted by $O_{s}-x_{s} y_{s} z_{s}$, where $O_{s}$ is the origin of the sub-reflector and $O_{s}-z_{s}$ coincides with the sub-reflector's axis and points to the main reflector. Here, points $O_{s}$ and $D_{s}$ are used to determine the subreflector's position and attitude. The ideal spherical coordinates of the measurement targets are marked as $\left(d_{a}^{s}, \theta_{a}^{s}, \varphi_{a}^{s}\right)$ in the instrument coordinate system, which can be easily calculated by the ideal design parameters.

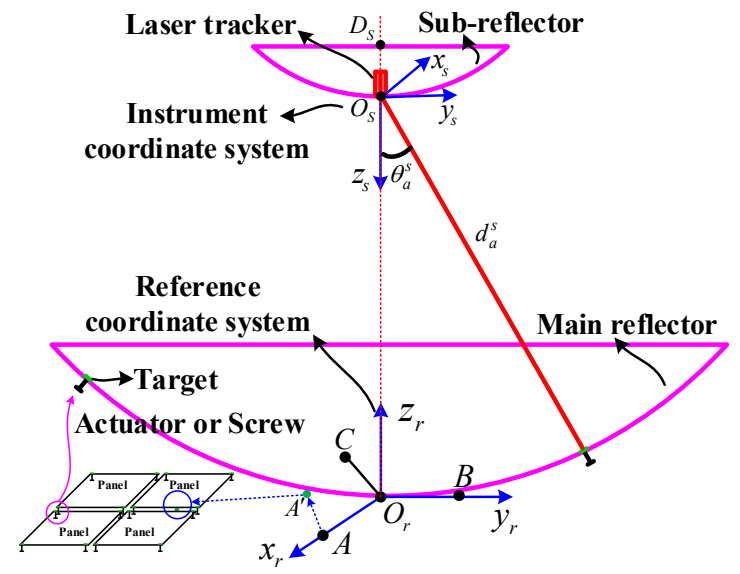

Fig. 3 Geometric diagram of the ideal dual-reflector telescope and laser measurement system
For an ideal telescope, because all the locations of the targets can be known in advance, the laser tracker can automatically find the targets by being given the angles $\left(\theta_{a}^{s}, \varphi_{a}^{s}\right)$. When the telescope is deformed under external environmental impacts, the targets will also slightly deviate from their ideal positions, and the measurement instrument will experience a rigid displacement when the sub-reflector is moved. In this case, the instrument should first be given the angles $\left(\theta_{a}^{s}, \varphi_{a}^{s}\right)$ and then should automatically search the measurement targets in a small area, which is possible because the deformations are usually very small.

However, in engineering, although there are no difficulties in installing the measurement instrument on the back of the sub-reflector, there are still some other problems that need to be further considered. For example, we should ensure that the measurement system does not affect the reflector's aperture field and that a suitable structure is designed, such as the wheel rail for a measurement at the edge of the sub-reflector, or the round door hole for a measurement at the centre of the sub-reflector. These points should be further considered by the structural designers and control designers and will not be discussed in this paper.

\section{Adjustment to the ideal location}

This section focuses on describing the adjustment process of the deformed reflectors to their ideal locations. As shown in Fig. 4, under the effects of environmental loads, the main reflector will be deformed, and the sub-reflector will be shifted slightly. Thus, the measurement instrument will also experience a slight deviation. In this case, the measured distance and the elevation angle are denoted by $d_{a}^{\prime s}$ and $\theta_{a}^{\prime s}$, respectively, where the superscript ' used in this paper denotes the quantities associated with the deformed telescope. Here, the rotations of the main reflector around the axis $O_{r}-z_{r}$ and the sub-reflector around the axis $O_{s} D_{s}$ are neglected, and as a result, the measured azimuth angle $\varphi_{a}^{\prime s}$ can be replaced by the ideal azimuth angle $\varphi_{a}^{s}$. Then, the measured spherical coordinates $\left(d_{a}^{\prime s}, \theta_{a}^{\prime s}, \varphi_{a}^{\prime s}\right)$ can be transformed into Cartesian coordinates $\left(x_{a}^{\prime s}, y_{a}^{\prime s}, z_{a}^{\prime s}\right)$, expressed as

$$
\left\{\begin{array}{c}
x_{a}^{\prime s}=d_{a}^{\prime s} \sin \theta_{a}^{\prime s} \cos \varphi_{a}^{\prime s} \\
y_{a}^{\prime s}=d_{a}^{\prime s} \sin \theta_{a}^{\prime s} \sin \varphi_{a}^{\prime s} \\
z_{a}^{\prime s}=d_{a}^{\prime s} \cos \theta_{a}^{\prime s}
\end{array}\right.
$$

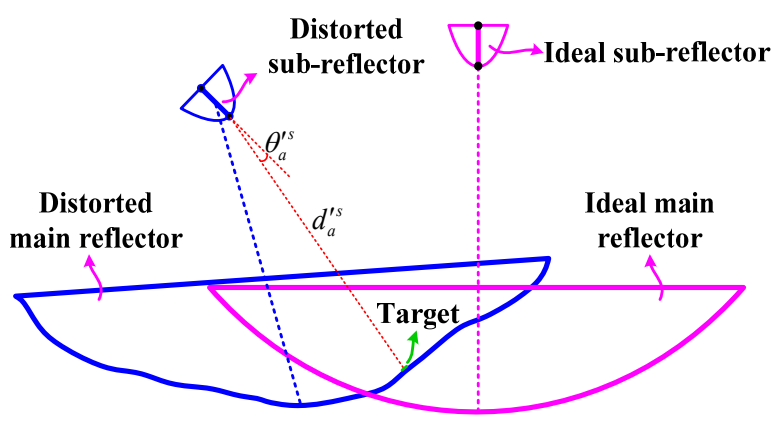

Fig. 4 Geometric diagram of the ideal dual-reflector telescope and deformed dual-reflector telescope 
Next, to determine the transformation relationship between the instrument coordinate system and the reference coordinate system, we need to select three points as reference points in the reference coordinate system, which are denoted by $A, B$ and $C$ in Fig. 3. It should be pointed out that there are two requirements for the reference points. The first requirement is that their deformation displacements should be much smaller than the telescope's deformations, which requires the locations to be near the centre of the main reflector. In this case, the best method is to separate the reference points from the main reflector through a suitable structural design to avoid the effect of the main reflector deformation and ensure that the reference points are not affected by the external environmental impacts, such as temperature and wind, through some protection measures and compensation technologies. As a result, the reference points' deformation can be approximated as zero. The second requirement is that the points $A, B, C$, and $O_{r}$, as shown in Fig. 3 , must not be coplanar to ensure that the transformation matrix between the instrument coordinate system and the reference coordinate system can be derived.

Here, the most important point is that the three reference points $A, B$, and $C$ in Fig. 3 are behind the main reflector, which means that the three points are out of sight of the laser tracker. It should be clarified that Fig. 3 is just a schematic for the locations of the reference points to show that the reference coordinate system can be derived by these three reference points. In fact, these reference points can be located anywhere as long as the two requirements mentioned above are met. Therefore, to ensure that the reference points can be measured by the laser tracker instead of other instruments to reduce the complexity of the measurement system, we can select the three points near the gap between the panels. For example, point $A$ can be replaced by point $A$, as shown in Fig. 3, and in this case, the laser tracker can measure point $A$ ' through the panel gap. In terms of engineering, this gap can be slightly modified for the measurement target installation if necessary.

For the reference points $A, B$, and $C$, similar to (4), the measured coordinate values can be described as,

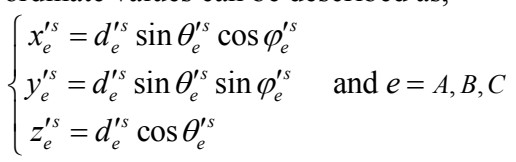

where $d_{e}^{\prime s}$ and $\theta_{e}^{\prime s}$ are the measured distance and the elevation angle, respectively, and the measured azimuth angle $\varphi_{e}^{\prime s}$ can be replaced by the ideal azimuth angle $\varphi_{e}^{s}$ for simplicity.

The relationship between the instrument coordinate system and the reference coordinate system includes two parts: a translation and a rotation. Obviously, the translation is determined by the coordinates of the origin of the instrument coordinate system in the reference coordinate system. Assume that the coordinates are $x_{O_{s}}^{\prime r}, y_{O_{s}}^{\prime r}$, and $z_{O_{s}}^{\prime r}$, and then the following equations hold

$$
\left\{\begin{array}{c}
\left\|\left(x_{A}^{r}, y_{A}^{r}, z_{A}^{r}\right)-\left(x_{O_{s}}^{\prime r}, y_{O_{s}}^{\prime r}, z_{O_{s}}^{\prime r}\right)\right\|=d_{A}^{\prime s} \\
\left\|\left(x_{B}^{r}, y_{B}^{r}, z_{B}^{r}\right)-\left(x_{O_{s}}^{\prime r}, y_{O_{s}}^{\prime r}, z_{O_{s}}^{\prime r}\right)\right\|=d_{B}^{\prime s} \\
\left\|\left(x_{C}^{r}, y_{C}^{r}, z_{C}^{r}\right)-\left(x_{O_{s}}^{\prime r}, y_{O_{s}}^{\prime r}, z_{O_{s}}^{\prime r}\right)\right\|=d_{C}^{\prime s} \\
z_{O_{s}}^{\prime r}>0
\end{array}\right.
$$

where $x_{e}^{r}, y_{e}^{r}$, and $z_{e}^{r}$ express the coordinates of the reference point $A$ in the reference coordinate system when the subscript $e$ is replaced by $A$, which is the same as the points $B$ and $C$. Obviously, the translations $x_{O_{s}}^{\prime r}, y_{O_{s}}^{\prime r}$, and $z_{O_{s}}^{\prime r}$ from the reference coordinate system to the instrument coordinate system can be determined by solving the following optimization model

$$
\begin{array}{ll}
\text { find } & x_{O_{s}}^{\prime r}, y_{O_{s}}^{\prime r}, z_{O_{s}}^{\prime r} \\
\min & \Delta d=\left(\left|\Delta d_{1}\right|+\left|\Delta d_{2}\right|+\left|\Delta d_{3}\right|\right) / 3 \\
\text { s.t. } & \Delta d_{1}=d_{A}^{\prime s}-\left\|\left(x_{A}^{r}, y_{A}^{r}, z_{A}^{r}\right)-\left(x_{O_{s}}^{\prime r}, y_{O_{s}}^{\prime r}, z_{O_{s}}^{\prime r}\right)\right\| \\
& \Delta d_{2}=d_{B}^{\prime s}-\left\|\left(x_{B}^{r}, y_{B}^{r}, z_{B}^{r}\right)-\left(x_{O_{s}}^{\prime r}, y_{O_{s}}^{\prime r}, z_{O_{s}}^{\prime r}\right)\right\| \\
& \Delta d_{3}=d_{C}^{\prime s}-\left\|\left(x_{C}^{r}, y_{C}^{r}, z_{C}^{r}\right)-\left(x_{O_{s}}^{\prime r}, y_{O_{s}}^{\prime r}, z_{O_{s}}^{\prime r}\right)\right\| \\
& z_{O_{s}}^{\prime r}>0
\end{array}
$$

Obviously, model (7) is a simple constrained nonlinear multivariable optimization problem, and in this paper, the "fmincon" function in MATLAB is directly applied to solve (7). The function "fmincon" has many optimization methods, such as the interior-point method, trust-region-reflective method, sequential quadratic programming (SQP) method, and active-set method, and the interior-point method is selected in the following simulations.

For the rotation, we denote the rotation matrix from the instrument coordinate system of the deformed telescope to the reference coordinate system by $\mathbf{T}_{s 2 r}^{\prime}$, and then the following equations hold

$$
\left\{\begin{array}{l}
\left(x_{A}^{r}, y_{A}^{r}, z_{A}^{r}\right)=\left(x_{A}^{\prime s}, y_{A}^{\prime s}, z_{A}^{\prime s}\right) \mathbf{T}_{s 2 r}^{\prime}+\left(x_{O_{s}}^{\prime r}, y_{O_{s}}^{\prime r}, z_{O_{s}}^{\prime r}\right) \\
\left(x_{B}^{r}, y_{B}^{r}, z_{B}^{r}\right)=\left(x_{B}^{\prime s}, y_{B}^{\prime s}, z_{B}^{\prime s}\right) \mathbf{T}_{s 2 r}^{\prime}+\left(x_{O_{s}}^{\prime r}, y_{O_{s}^{\prime}}^{\prime r}, z_{O_{s}}^{\prime r}\right) \\
\left(x_{C}^{r}, y_{C}^{r}, z_{C}^{r}\right)=\left(x_{C}^{\prime s}, y_{C}^{\prime s}, z_{C}^{\prime s}\right) \mathbf{T}_{s 2 r}^{\prime}+\left(x_{O_{s}}^{\prime r}, y_{O_{s}}^{\prime r}, z_{O_{s}}^{\prime r}\right)
\end{array}\right.
$$

By solving (8), we can obtain

$$
\mathbf{T}_{s 2 r}^{\prime}=\left[\begin{array}{lll}
x_{b(A)}^{\prime s} & y_{b(4)}^{\prime s} & z_{b(A)}^{\prime s} \\
x_{b(B)}^{(s)} & y_{b(B)}^{\prime s} & z_{b(B)}^{\prime s} \\
x_{b(C)}^{(s)} & y_{b(C)}^{\prime s} & z_{b(C)}^{\prime s}
\end{array}\right]^{-1}\left[\begin{array}{lll}
x_{A}^{r}-x_{O_{s}}^{\prime r} & y_{A}^{r}-y_{O_{s}}^{\prime r} & z_{A}^{r}-z_{O_{s}}^{\prime r} \\
x_{B}^{r}-x_{O_{s}}^{\prime r} & y_{B}^{r}-y_{O_{s}^{\prime}}^{\prime r} & z_{B}^{r}-z_{O_{s}}^{\prime r} \\
x_{C}^{r}-x_{O_{s}}^{\prime r} & y_{C}^{r}-y_{O_{s}}^{\prime r} & z_{C}^{r}-z_{O_{s}}^{\prime r}
\end{array}\right] \text { (9) }
$$

With $\mathbf{T}_{s 2 r}^{\prime}$, we can easily obtain the coordinates of the measurement targets in the reference coordinate system, which can be written as

$$
\left(x_{a}^{\prime r}, y_{a}^{\prime r}, z_{a}^{\prime r}\right)=\left(x_{a}^{\prime s}, y_{a}^{\prime s}, z_{a}^{\prime s}\right) \mathbf{T}_{s 2 r}^{\prime}+\left(x_{O_{s}}^{\prime r}, y_{O_{s}}^{\prime r}, z_{O_{s}}^{\prime r}\right)
$$

Therefore, we can easily derive the adjustments of the actuators according to the displacements in the $z$-direction, as indicated in Fig. 5, and the expression is

$$
\Delta n_{a}=\left(z_{a}^{r}-z_{a}^{\prime r}\right) / \chi_{a}
$$

in which $\chi_{a}=\operatorname{dot}\left\{(0,0,1) \cdot \tilde{\boldsymbol{n}}_{a}\right\}$ and $\tilde{\boldsymbol{n}}_{a}$ is the unit normal vector of the main reflector.

The position and posture of the sub-reflector can be determined by the coordinates of the points $O_{s}$ and $D_{s}$. Fig. 6 shows the locations of the sub-reflector before and after the deformation, and the points $O_{s}^{\prime}$ and $D_{s}^{\prime}$ are the corresponding locations of points $O_{s}$ and $D_{s}$ after the deformation. Then, it is clear that the key to determining the sub-reflector's adjustment is to obtain the coordinates of the points $O_{s}, D_{s}$, $O_{s}^{\prime}$, and $D_{s}^{\prime}$ in the reference coordinate system. We know that the points $O_{s}$ and $D_{s}$ are on the ideal sub-reflector, so their coordinates can be easily obtained by the ideal geometric shape shown in Fig. 3 and are denoted by $\left(x_{O_{s}}^{r}, y_{O_{s}}^{r}, z_{O_{s}}^{r}\right)$ and 
$\left(x_{D_{s}}^{r}, y_{D_{s}}^{r}, z_{D_{s}}^{r}\right)$, respectively. For the point $O_{s}^{r}$, its coordinate is derived by solving (7) in the preceding part, and the corresponding adjustment can be expressed as

$$
\left(\Delta x_{O_{s}}, \Delta y_{O_{s}}, \Delta z_{O_{s}}\right)=\left(x_{O_{s}}^{r}, y_{O_{s}}^{r}, z_{O_{s}}^{r}\right)-\left(x_{O_{s}}^{\prime r}, y_{O_{s}}^{\prime r}, z_{O_{s}}^{\prime r}\right)
$$

For the point $D_{s}^{\prime}$, its coordinate can be derived through the translation between the instrument coordinate system and the reference coordinate system, and the expression is

$$
\left(x_{D}^{\prime r}, y_{D}^{\prime r}, z_{D}^{\prime r}\right)=\left(0,0, z_{D}^{s}\right) \mathbf{T}_{s 2 r}^{\prime}+\left(x_{O_{s}}^{\prime r}, y_{O_{s}}^{\prime r}, z_{O_{s}}^{\prime r}\right)
$$

Then, the corresponding adjustment can be expressed as

$$
\left(\Delta x_{D}, \Delta y_{D}, \Delta z_{D}\right)=\left(x_{D_{s}}^{r}, y_{D_{s}}^{r}, z_{D_{s}}^{r}\right)-\left(x_{D}^{\prime r}, y_{D}^{\prime r}, z_{D}^{\prime r}\right)
$$

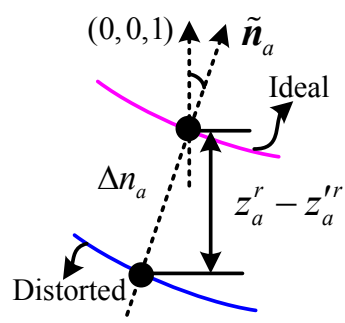

Fig. 5 Geometric diagram of the adjustment calculation for the main reflector

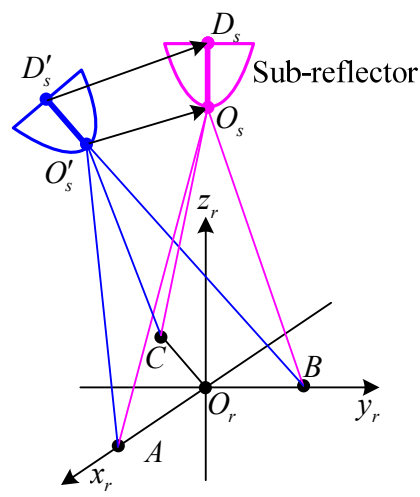

Fig. 6 Geometric diagram of the adjustment calculation for the deformed sub-reflector

Until now, we have derived the adjustments for both the main reflector and the sub-reflector through (11), (12), and (14). As the telescope operates, the measurement system can continue working and the main reflector and the subreflector can always be adjusted to their ideal locations.

\section{Adjustment to the best fitting location}

In engineering, the best fitting reflector shown in Fig. 7 is always selected to measure the surface accuracy instead of an ideal reflector. The best fitting accuracy is more reasonable and accurate for characterizing the telescope's precision. In this case, the sub-reflector should be moved to the focus of the best fitting reflector, and at the same time, the adjustments of the main reflector will be significantly reduced. It should be noted that the best fitting reflector mentioned here is different from the traditional best fitting paraboloid. In this paper, an ideal reflector is directly adopted to fit the deformed reflector, so the shape of the best fitting reflector is the same as that of the ideal reflector. The reason for this is that the main reflector is usually a shaped reflector, which is not an ideal paraboloid. To ensure that the best fitting reflector and the sub-reflector can match each other very well, the best fitting reflector must be identical to the ideal reflector. To calculate the adjustments, the best fitting reflector should be determined first. As shown in Fig. 7, when the telescope is deformed, we assume that there is one imaginary best matched reflector that matches the real sub-reflector perfectly. Then, the best fitting reflector can be obtained through a rotation and translation of the best matched reflector, and the real sub-reflector will also be moved to the new focus with the same rotation and translation.

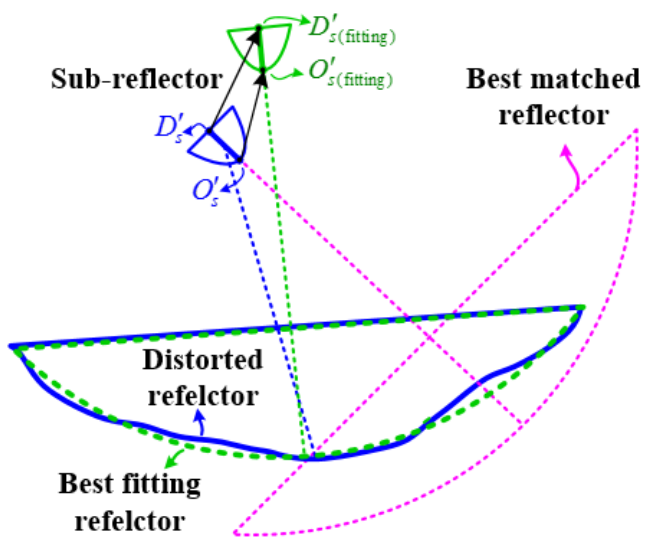

Fig. 7 Geometric diagram of the best fitting process

According to Fig. 3, the ideal coordinates of the measurement targets in the instrument coordinate system can be easily identified and are expressed as $\left(x_{a}^{s}, y_{a}^{s}, z_{a}^{s}\right)$, where $a=1,2, \cdots, N$. For the best matched reflector in Fig.7, its coordinates in the instrument coordinate system for the real reflector are the same as the ideal coordinates in the instrument coordinate system for the ideal reflector and can also be expressed as $\left(x_{a}^{s}, y_{a}^{s}, z_{a}^{s}\right)$. Then, the corresponding four-dimensional coordinates can be described as

$$
\mathbf{V}_{\text {ideal }}=\left[\begin{array}{cccc}
x_{1}^{s} & y_{1}^{s} & z_{1}^{s} & 1 \\
x_{2}^{s} & y_{2}^{s} & z_{2}^{s} & 1 \\
\vdots & \vdots & \vdots & \vdots \\
x_{N}^{s} & y_{N}^{s} & z_{N}^{s} & 1
\end{array}\right]
$$

Here, it should be pointed out that because the translation and rotation are performed simultaneously, fourdimensional coordinates are adopted to obtain the fourdimensional transformation matrix, in which the first three dimensions correspond to the rotation transformation and the fourth dimension corresponds to the translation transformation.

Similarly, according to (4), the four-dimensional coordinates of the measurement targets for the deformed reflector in the instrument coordinate system can be described as

$$
\mathbf{V}_{\text {distortion }}^{\prime}=\left[\begin{array}{cccc}
x_{1}^{\prime s} & y_{1}^{\prime s} & z_{1}^{\prime s} & 1 \\
x_{2}^{\prime s} & y_{2}^{\prime s} & z_{2}^{\prime s} & 1 \\
\vdots & \vdots & \vdots & \vdots \\
x_{N}^{\prime s} & y_{N}^{\prime s} & z_{N}^{\prime s} & 1
\end{array}\right]
$$

Similar to the concept of the best fitting paraboloid, 
for the deformed reflector with a rigid-body displacement and a flexible deformation, a transformation matrix is needed to ensure that the real reflector approximates the best matched reflector through the coordinate transformation in the least square sense. This fitting process can be expressed by the following equation

$$
\mathbf{V}_{\text {distortion }}^{\prime} \approx \mathbf{V}_{\text {ideal }} T_{\text {fitting }}
$$

in which $\mathbf{T}_{\text {fitting }}$ is the four-dimensional transformation matrix that can be derived as

$$
\mathbf{T}_{\text {fitting }} \approx\left(\mathbf{V}_{\text {ideal }}^{\mathrm{Tr}} \mathbf{V}_{\text {ideal }}\right)^{-1} \mathbf{V}_{\text {ideal }}^{\mathrm{Tr}} \mathbf{V}_{\text {distortion }}^{\prime}
$$

where the superscript $\mathrm{Tr}$ is the transposition operator.

Next, we further derive the adjustments of the main reflector and the sub-reflector with the help of the transformation matrix $\mathbf{T}_{\text {fiting }}$.

For the main reflector, the adjustments are determined by the difference between the coordinates of the measurement targets for the best fitting reflector and those for the deformed reflector. Obviously, the coordinates of the measurement targets for the best fitting reflector can be easily obtained by the following equation

$$
\mathbf{V}_{\text {fitting }}^{\prime}=\left\langle\mathbf{V}_{\text {ideal }} \mathbf{T}_{\text {fitting }}\right\rangle_{(N \times 3)}
$$

in which $\langle\bullet\rangle_{(N \times 3)}$ stands for the operation of extracting the elements in the first $N$ rows and the first three columns of the bracketed matrix; in addition, the data in each row of $\mathbf{V}_{\text {fitting }}^{\prime}$ represent the coordinates of the measurement targets for the best fitting reflector, which for simplicity, can be expressed as $\left(x_{a \text { (fiting) }}^{\prime s}, y_{a(\text { (fiting) }}^{\prime s}, z_{a \text { (fiting) }}^{\prime s}\right)$. Thus, the adjustments of the actuators can be described as

$$
\Delta n_{a \text { (fiting) }}=\operatorname{sign}\left(\Delta n_{a(\text { fiting })}\right) \cdot \operatorname{norm}\left[\tilde{\boldsymbol{n}}_{a}^{\prime}\left(z_{a(\text { fiting })}^{\prime s}-z_{a}^{\prime s}\right) /\left(\left.\tilde{\boldsymbol{n}}_{a}^{\prime}\right|_{z}\right)\right]
$$

where the operator norm[ $[\bullet]$ represents the length of the bracketed vector, the operator $(\bullet)$, represents a vector's zcomponent, the operator $\operatorname{sign}(\bullet)$ is the sign function, and $\tilde{\boldsymbol{n}}_{a}^{\prime}$ is the unit normal vector of the best fitting reflector:

$$
\begin{gathered}
\operatorname{sign}\left(\Delta n_{a(\text { fitting })}\right)=\left\{\begin{array}{cc}
-1 & z_{a(\text { fitting })}^{\prime s}<z_{a}^{\prime s} \\
1 & z_{a(\text { fitting })}^{\prime}>z_{a}^{\prime s}
\end{array}\right. \\
\tilde{\boldsymbol{n}}_{a}^{\prime \operatorname{Tr}}=\tilde{\boldsymbol{n}}_{a}^{\mathrm{Tr}}\left\langle\mathbf{T}_{\text {fitting }}\right\rangle_{(3 \times 3)}
\end{gathered}
$$

For the sub-reflector, the coordinates of the points $O_{s}^{\prime}$ and $D_{s}$ in the instrument coordinate system can be expressed as $(0,0,0)$ and $\left(0,0, z_{O_{s}}^{r}-z_{D_{s}}^{r}\right)$, respectively, and can be further rewritten as four-dimensional coordinates as $(0,0,0,1)$ and $\left(0,0, z_{O}^{r}-z_{D}^{r}, 1\right)$. Then, the coordinates of points $O_{s(\text { fititing })}^{\prime}$ and $D_{s(\text { fitting })}^{\prime}$ in the instrument coordinate system can be derived by the following equation

$$
\begin{aligned}
& \left(x_{O_{s(\text { fftiting })}^{\prime s}}^{\prime s}, y_{O_{s(\text { fititing })}^{\prime s}}^{\prime s}, z_{S_{s(\text { fitting })}^{\prime s}}^{\prime s}\right)=\left\langle(0,0,0,1) \mathbf{T}_{\text {fitting }}\right\rangle_{(1 \times 3)} \\
& \left(x_{D_{s(\text { fiting })}}^{\prime s}, y_{D_{s(\text { fititug })}^{\prime s},}^{\prime s}, z_{D_{s(\text { fiting })}^{\prime s}}^{\prime s}\right)=\left\langle\left(0,0, z_{O_{s}}^{r}-z_{D_{s}}^{r}, 1\right) \mathbf{T}_{\text {fitting }}\right\rangle_{(1 \times 3)}
\end{aligned}
$$

Thus, the adjustments of the sub-reflector in the instrument coordinate system can be expressed as

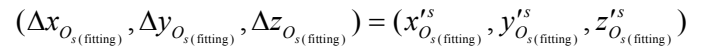

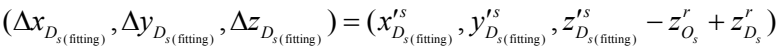

Thus far, the adjustment process for the best fitting reflector has been presented. The best fitting method can guarantee that the telescope is always kept at the location of the best fitting reflector during operation, which is the same as the ideal reflector to guarantee a good match with the subreflector.

Compared with the method presented in Section 4, the difference is that the best fitting method requires no reference points, which will reduce the complexity of the measurement system and the adjustment amounts will also be reduced. However, in engineering, adjusting the panels to their ideal locations is a common practice. Therefore, the two presented methods are reasonable, and simulations and comparisons will be presented for a comparison in the following section.

\section{Simulations}

In this section, to assess the performance of the proposed methods, a dual-reflector telescope is adopted for the verification, where the diameters of the shaped main reflector and the sub-reflector are $35 \mathrm{~m}$ and $2.4 \mathrm{~m}$, respectively. The focal length of the main reflector is 10.83 $\mathrm{m}$, the magnification $M$ of the focal length is 6.846, the working frequency is $10 \mathrm{GHz}$, and the aperture amplitude distribution in equation (3) is expressed as [27]

$$
F(r, \phi)=0.315+(1-0.315) \cdot\left(1-r^{2}\right)^{1.5}
$$

Fig. 8 presents the deformation nephogram of the main reflector when the telescope is pointing horizontally; the rigid-body translation of the sub-reflector is $-6.315 \mathrm{~mm}$ along the $Y$ axis and the rigid-body rotation of the sub-reflector is $0.0012 \mathrm{rad}$ around the $X$ axis. In this case, we assume that all the vertices of the panels are adjustable with the actuators, and then the two methods presented in sections 4 and 5 will be adopted to calculate the adjustments of the main reflector and the sub-reflector to verify their validity.

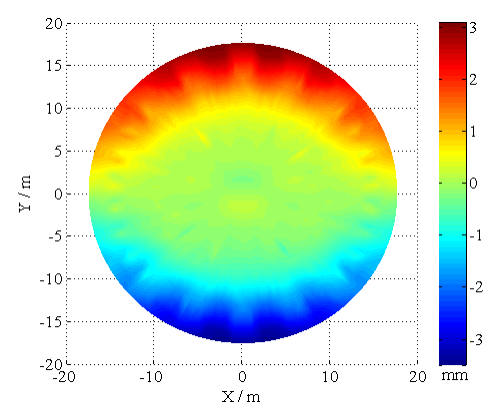

Fig. 8 Deformation nephogram of the main reflector when pointing horizontally

\subsection{Method A: Adjustment to the ideal location}

In this simulation, the coordinates of the reference points in the reference coordinate system are selected as (1.7, $0,0.066),(0,1.7,0.066)$, and $(-1.47,-0.85,0.066)$, and the coordinates of points $O_{s}$ and $D_{s}$ are $(0,0,10.882)$ and $(0,0$, 10.482), respectively. Here, the units are metres. It should be noted that the reference points are randomly selected near the centre of the main reflector. Then, the adjustments can be easily derived according to (11).

Fig. 9 presents the deformations in z-direction of the vertices of the panels equipped with actuators before and after the adjustment. The curve termed "Before Adjustment" 
represents the deformations before the adjustment, and the curve termed "Case 1" is the deformations after the adjustment. Obviously, the deformations are greatly reduced and the deformation range is narrowed from approximately $\pm 3.5 \mathrm{~mm}$ to $\pm 0.5 \mathrm{~mm}$, which means that the electromagnetic performance will be greatly improved according to the Ruze formula or its modified form [27-28]. Fig. 9 also clearly indicates that method A is not effective for relatively small deformations, such as the deformations of the first 200 vertices. The reason is that the rotation matrix derived by (9) from the instrument coordinate system to the reference coordinate system is not exact and will result in system errors. Fig. 10 shows the deformation nephogram of the main reflector after the adjustment and clearly shows that the distribution of the residual errors is regular, which is caused by the inexact rotation matrix. To improve the precision, we need to increase the number of reference points to obtain an optimal rotation matrix in the least-squares sense.

To examine the influence of the three reference points on the adjustment accuracy, the coordinates of the reference points in the reference coordinate system are reselected as (5.7, 0, 0.742), (0, 5.7, 0.742), and (-5.47, -2.05, 0.779), and the corresponding result is plotted in Fig. 9 and termed "Case 2 ". Compared with the previous reference points, the new points are farther away from the centre, and their distances between each other are longer.

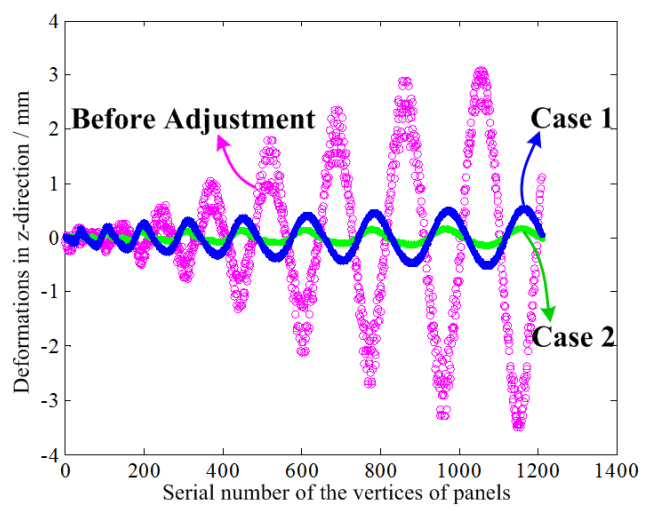

Fig. 9 Corner deformations of the panels in the z-direction for method $A$

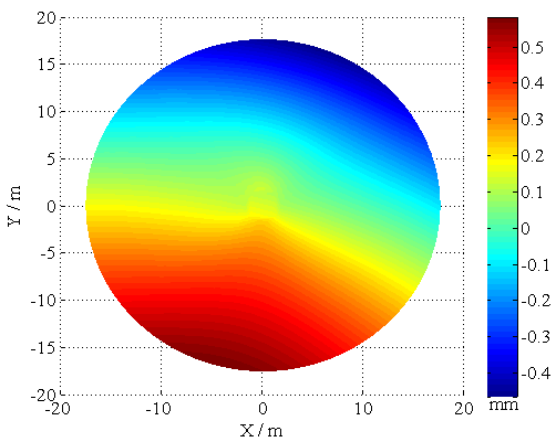

Fig. 10 Deformation nephogram of main reflector after the adjustment

Obviously, in this case, the adjustment accuracy increases and the deformation range is narrowed down from approximately $\pm 3.5 \mathrm{~mm}$ to $\pm 0.2 \mathrm{~mm}$. The reason is that the rotation matrix derived by (9) will be more accurate. It should be pointed out that the difficulty in the design of the support structures and the reference point's deformation should be considered when determining the locations. Fig. 11 shows the structures of the Nanshan $26 \mathrm{~m}$ telescope and the Effelsberg $100 \mathrm{~m}$ telescope. The common features are that both have one centre hub and one elevation axis. For the larger telescope, generally, the centre hub is compact and massive and made of steel, and the whole backup structure is supported by the rigid fork or alidade at points A and B. Due to the high stiffness of the centre hub, the deformation will be very small, which is why the sub-reflector supporting legs are generally connected to the centre hub at points A, B, C, and D. Therefore, the reference points can also be located at the panel gaps over the points $\mathrm{A}, \mathrm{B}, \mathrm{C}$, and $\mathrm{D}$, or the gaps between panels and sub-reflector supporting legs, or other locations within the centre hub. Generally, the distance between the reflector panel and main beam of the centre hub is relatively small, which will reduce the difficulty of designing the supporting structures of the reference points. To avoid the effect of the deformation of the backup structure, the reference point's supporting structure should be an independent sub-truss supported by the centre hub at points $\mathrm{A}, \mathrm{B}, \mathrm{C}$, or D.
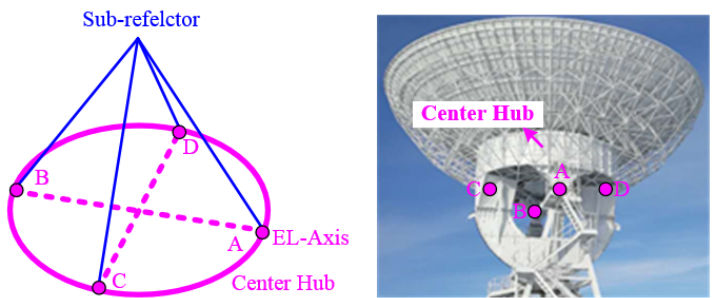

(a)

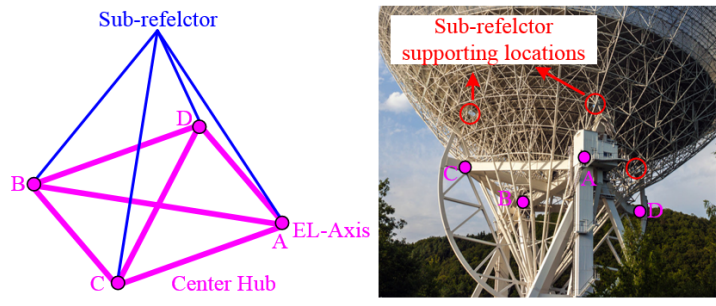

(b)

Fig. 11 Support structures of the telescopes

(a) Nanshan $26 \mathrm{~m}$ telescope, China; (b) Effelsberg $100 \mathrm{~m}$ telescope, Germany

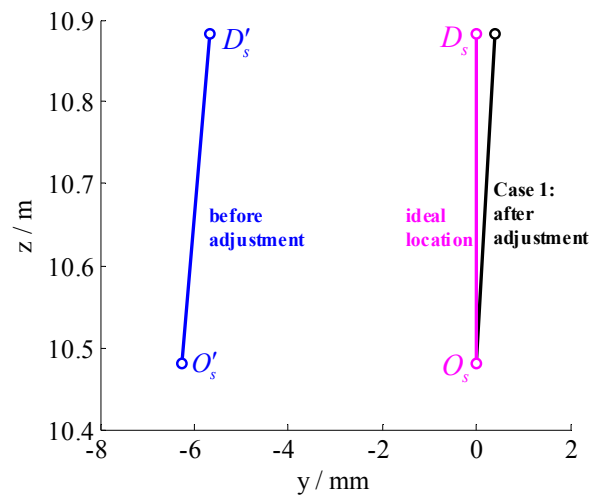

Fig. 12 Adjustments of the sub-reflector in the $y$-direction 
Next, the adjustment process of the sub-reflector is discussed. When the telescope is pointing horizontally, the sub-reflector will be deformed and the displacements are mainly in the y-direction because of the symmetry of the telescope's structure. Thus, Fig. 12 only shows the adjustments in the y-direction. In Fig.12, the line segment termed "before adjustment" is the axis of the distorted subreflector, and the line segment termed "ideal location" is the axis of the ideal sub-reflector. Fig.6 shows the adjustment process, the purpose of the adjustment process is to move the distorted sub-reflector to the ideal location. Here, the axis of the sub-reflector is adopted to indicate its location and posture. Fig. 12 presents the location in the y-direction of the axis of the sub-reflector after the adjustment, and the axis is termed "after adjustment". Obviously, the sub-reflector can be accurately moved to the ideal location, but due to the adjustment error of point $D_{s}$, there still exists a small angle displacement after the adjustment.

Fig. 13 shows the patterns before and after the adjustment of the main reflector and the sub-reflector to the ideal location, and a comparison with the ideal pattern shows that the electromagnetic performance is greatly improved, which suggests that method $\mathrm{A}$ is effective.

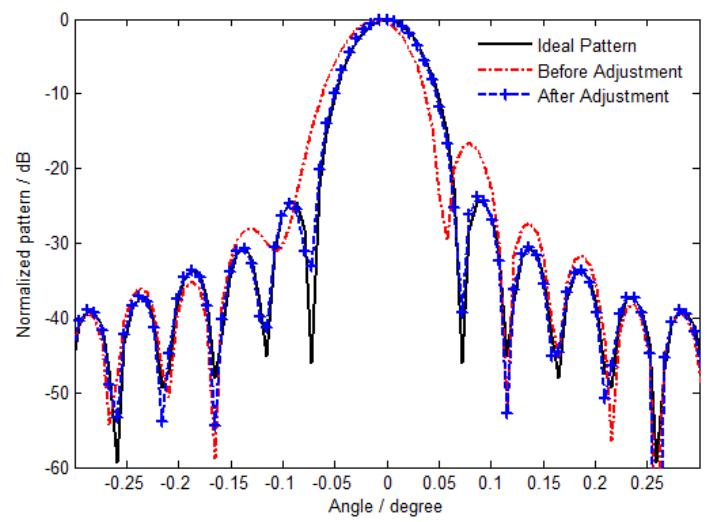

Fig. 13 Pattern comparison (adjustment to the ideal location)

\subsection{Method B: Adjustment to the best fitting location}

In this simulation, the adjustment to the best fitting location is discussed. According to (20), (25), and (26), the adjustments of the main reflector and the sub-reflector can be directly obtained. Fig. 14 presents the corner deformations of the panels in the z-direction before and after the adjustment of the main reflector and the sub-reflector. The curve termed "Before Adjustment" represents the deformations before the adjustment. The curve termed "Adjust Sub-reflector" represents the residual errors when the sub-reflector is moved to the focus of the best fitting reflector and the main reflector is not adjusted. The curve termed "Adjust Main Reflector" is the corresponding residual errors when the main reflector and the sub-reflector are adjusted. Obviously, the following conclusions can be derived.

1) The sub-reflector adjustment can greatly reduce the reflector's surface rms error, and the error range is narrowed from approximately $\pm 3.5 \mathrm{~mm}$ to $\pm 1 \mathrm{~mm}$, which will greatly improve the telescope's electromagnetic performance according to the Ruze formula.
2) The most apparent feature is that the large errors are greatly reduced and the small errors are slightly increased for the sub-reflector adjustment. Fig. 15 shows the distribution of the residual errors; compared with that presented in Fig. 8, the distribution is more uniform, which will greatly improve the telescope's pointing accuracy.

3) When the sub-reflector and the main reflector are adjusted, the residual errors are nearly zero in an ideal situation. The main reason is that the adjustments of method $B$ are smaller than those of method A, which will make it easier to adjust the panels to specific locations.

4) The adjustments of the actuators calculated by method B are smaller than those derived by method A, which is clearly shown in Fig. 16; at the same time, the adjustments are more uniform, which is helpful for the adjustment of the actuators.

5) Fig. 17 directly shows the patterns before and after the adjustment of the main reflector and the sub-reflector to the best fitting location. Obviously, just adjusting the subreflector to the best fitting location will also result in a great improvement, which shows that method B is also suitable for the traditional telescopes.

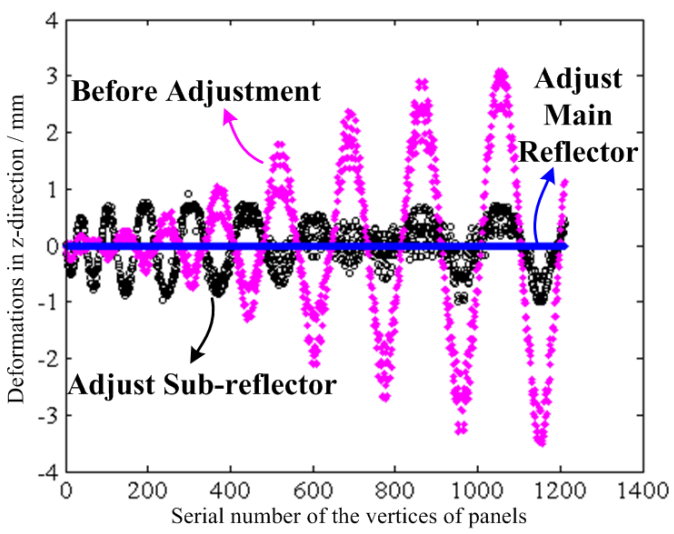

Fig. 14 Corner deformations of the panels in the z-direction for method $B$

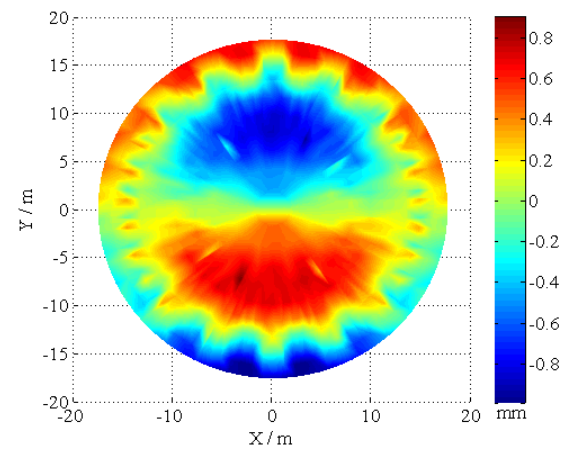

Fig. 15 Deformation nephogram of the main reflector after the sub-reflector adjustment 


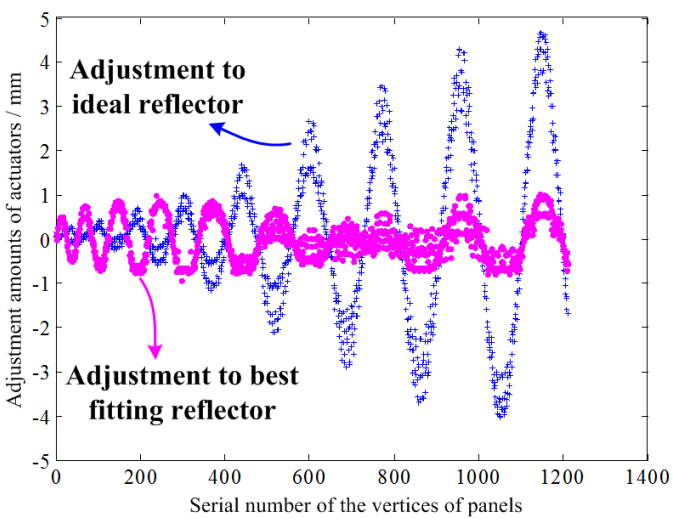

Fig. 16 Comparison of the adjustment amounts for methods $A$ and $B$

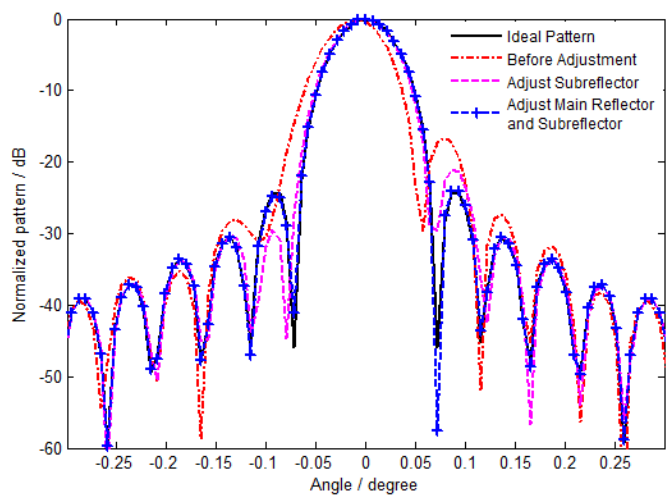

Fig. 17 Pattern comparison (adjustment to the best fitting location)

Table 1 Electromagnetic performance comparison of the patterns presented in Fig. 13 and Fig. 17.

\begin{tabular}{ccccc}
\hline Cases & GL/dB & L-FSLL/dB & R-FSLL/dB & PE/ \\
\hline Case 1 & 0 & -24.41 & -24.41 & 0 \\
Case 2 & 0.17 & -27.82 & -16.42 & -0.01 \\
Case 3 & 0.01 & -24.69 & -23.82 & $\approx 0$ \\
Case 4 & 0.02 & -29.46 & -20.99 & $\approx 0$ \\
Case 5 & $\approx 0$ & -24.81 & -24.06 & $\approx 0$ \\
\hline
\end{tabular}

To further compare the electromagnetic performance after the panel adjustment for the presented two methods, the main electromagnetic parameters of the patterns shown in Fig. 13 and Fig. 17 are listed in Table 1. Here, the gain loss (GL), the left first side lobe level (L-FSLL), the right first side lobe level (R-FSLL), and the pointing error (PE) are considered. "Case 1" and "Case 2" represent the cases where the parameters are calculated for the ideal reflector and the distorted reflector, respectively, and the data correspond to the patterns termed "Ideal Pattern" and "Before Adjustment", respectively. "Case 3", "Case 4", and "Case 5" denote the quantities associated with the patterns termed "After Adjustment" in Fig. 13 for method A, "Adjust Sub-reflector" in Fig. 17 for method B, and "Adjust Main reflector and Subreflector" in Fig. 17 for method B, respectively. Obviously, the PEs are almost zero, and the FSLLs are close to the ideal data, and the GL is reduced from $0.17 \mathrm{~dB}$ to $0.01 \mathrm{~dB}$ and 0 $\mathrm{dB}$ for method $\mathrm{A}$ and method $\mathrm{B}$ respectively. The GL of 0.01 $\mathrm{dB}$ for method $\mathrm{A}$ is caused by the adjustment errors of the main reflector due to the longer adjustments shown in Fig. 9 and the angle error of the sub-reflector shown in Fig. 12. Regardless, the performance improvement is evident for both of the proposed methods. A comparison with Case 2 and Case 4 further shows that method $\mathrm{B}$ is also suitable for the traditional telescope.

The above results and discussions clearly show that method A and method B are both effective in reducing the surface rms errors of the main reflector and the rigid body displacements of the sub-reflector, which will greatly improve the telescope's electromagnetic performance in terms of gain and pointing accuracy. The comparison between method A and method B also shows that method B may be more suitable for engineering applications. Method B can be applied to both the active main reflector and the traditional telescope, and there is no need to select several reference points, which will reduce the complexity of the measurement system. In addition, the adjustments of method $\mathrm{B}$ are smaller and more uniform than those of method A, which will be helpful for the adjustment of the actuators. However, it should be pointed out that telescope's mechanical axis and electric axis will always coincide with each other for method A, which may not be achieved by method B. Thus, telescope designers can choose method A or B according to the actual engineering requirements.

In engineering, measurement accuracy and surface control error are two important aspects that should be considered. The surface control error depends on the precision of the actuator and adjustment calculation error due to the change in the normal vector of the deformed reflector. These two kinds of errors are relatively small and do not affect the adjustment. For the measurement accuracy, in this paper, good simulation results are obtained with a precise position measurement accuracy. The laser tracker is installed on the sub-reflector to measure the target's coordinate; when considering its measurement accuracy, the measured coordinate will be imprecise, and the scale of the coordinate error will be the same as the laser tracker's measurement accuracy. Current laser trackers, such as the API or Faro laser trackers, have a very high ranging precision. For example, the distance measurement precision of the Faro vantage laser tracker can be as high as $16 \mathrm{um}+0.8 \mathrm{um} / \mathrm{m} \times d$, where $d$ is the measurement distance and the displacement error caused by the angle measurement error for this laser tracker is approximately $20 \mathrm{um}+5 \mathrm{um} / \mathrm{m} \times d$. Thus, for a measurement distance of $20 \mathrm{~m}$, the largest measurement error for a distance measurement and angle measurement will be less than 0.032 $\mathrm{mm}$ and $0.12 \mathrm{~mm}$, respectively. Of course, this measurement accuracy may restrict the application of the presented method in some sub-millimetre wave telescopes, such as the LMT 50 $\mathrm{m}$ telescope. However, for most other large telescopes, especially for the millimetre telescopes, the measurement error is very small compared with the structural deformations. Therefore, the presented method will still significantly improve the reflector's surface accuracy and electromagnetic performance, and the adjustment will not be affected by small measurement errors. In engineering, we can also adopt an instrument with a higher measurement precision if necessary, or we can adopt more than one instrument to measure distance due to the high distance measurement precision. However, this requires further study. 


\section{Conclusion}

This work presents an initial surface adjustment strategy to realize an automation adjustment for a large radio telescope with adjustable dual reflectors. In the presented strategy, two different methods of adjusting the deformed telescope to the ideal design location and to the best fitting location are derived and discussed through several simulations. The results clearly show that the two methods can both be very effective in reducing the surface errors and improving the electromagnetic performance in terms of gain, side lobe level, and pointing error. The best fitting method is more suitable for an implementation in engineering because of its small adjustments and lack of requirements for reference points. In fact, there are still some problems that need to be solved for the implementation of the presented method in engineering applications. For example, the installation and operation of the measuring system should not affect the telescope's electromagnetic performance. The proposed strategy is presented as a reference for the design of automation surface adjustment. In terms of engineering, much more work is needed to realize closed-loop control in a real-time manner, and further studies are still needed to improve the telescope's performance.

\section{Acknowledgements}

The authors would like to express their gratitude to the staff of the Key Laboratory of Electronic Equipment Structure Design, Ministry of Education, Xidian University, China, and the staff of the Xinjiang Astronomical Observation, Chinese Academy of Sciences, Urumqi 830011, China, for their discussions.

This project was supported in part by the National Natural Science Foundation of China No. 51805399, the Shaanxi Natural Science Basic Research Project No. 2019JQ144, the Fundamental Research Funds for the Central Universities No. JB180403, and the CAS "Light of West China" Program No. 2017-XBQNXZ-B-023 and No. 2017XBQNXZ-B-024.

\section{References}

[1] Rahmat-Samii Y., Haupt R. L.: 'Reflector antenna developments: a perspective on the past, present, and future', IEEE Antennas and Propagation Magazine, 2015, 57, (2), pp. 85-95

[2] Ukita N., Ezawa H., Ikenoue B., et al.: 'Thermal and wind effects on the azimuth axis tilt of the ASTE $10-\mathrm{m}$ antenna', Publications of the National Astronomical Observatory of Japan, 2017, 10, pp: 25-33

[3] Greve A., Morris D.: 'The beam pattern of reflector antennas with buckled panels', IEEE Transactions on Antennas and Propagation, 2010, 58, (3), pp. 959-962

[4] Dong J., Zhong W. Y., Wang J. Q.: 'Correcting gravitational deformation at the Tianma radio telescope', IEEE Trans. Antennas Propag., 2018, 66, (4), pp. 20442048

[5] Sinton S., Rahmat-Samii Y.: 'Random surface error effects on offset cylindrical reflector antennas', IEEE Trans. Antennas Propag., 2003, 51, (6), pp. 1331-1337

[6] Duan B. Y., Wang C. S.: 'Reflector antenna distortion analysis using MEFCM', IEEE Trans. Antennas Propag., 2009, 57, (10), pp. 3409- 3413
[7] Paolo Rocca, Nicola Anselmi, Andrea Massa: 'Interval arithmetic for pattern tolerance analysis of parabolic reflectors', IEEE Trans. Antennas Propag., 2014, 62, (10), pp. 4952-4960

[8] Lian P. Y., Duan B. Y., Wang W., et al.: 'Effects of Nonuniform Surface Errors along the Radius on Reflector's Radiation Characteristic and Its Quality Evaluation', IEEE Trans. Antennas Propagat., 2015, 63, (5), pp. 2312-2316

[9] Ban Y., Duan B. Y., Wang C. S., et al.: 'Homology Parameters for Large Axisymmetric Shaped DualReflector Antennas', IEEE Trans. Antennas Propag., 2017, 65, (7), pp. 3392-3398

[10] S. Von Hoerner.: 'Homologous deformations of tiltable telescopes', ASCE Int. J. Struct. Division, 1976, 93, (5), pp. $461-486$

[11] S. Von Hoemer, Wong W.: 'Gravitational deformation and astigmatism of tiltable radio telescopes', IEEE Trans. Antennas Propag., 1975, 23, (5), pp. 689-695

[12] Liu J., Hollaway L.: Integrated structure-electromagnetic optimization of large reflector antenna systems', Struct. Multidisc. Optim., 1998, 16, (1), pp. 29-36

[13] Wang C. S., Duan B. Y., Qiu Y. Y.: 'On distorted surface analysis and multidisciplinary structural optimization of large reflector antennas', Structural and Multidisciplinary Optimization. 2007, 33, (6), pp. 519528

[14] Aderman H., Padula S.: 'Integrated thermal-structuralelectromagnetic design optimization of large space antenna reflectors', NASA Technical Memorandum 87713, Langley Research Center, Hampton, Virginia, 1986

[15]Lian P. Y., Duan B. Y., Wang W., et al.: 'A Pattern Approximation Method for Distorted Reflector Antennas Using Piecewise Linear Fitting of the Exponential Error Term', IEEE Trans. Antennas Propag., 2015, 63, (10), pp. 4546-4551

[16] Ryohei Kawabe, Kotaro Kohno, Yoichi Tamura, et al.: 'New 50-m-class single dish telescope: Large Submillimeter Telescope (LST)', Astrophysics, 2017, pp: 301-312

[17] Schloerb F. P., Sanchez D., Narayanan G., et al.: 'Calibration and Operation of the Active Surface of the Large Millimeter Telescope', Proc. of SPIE, 2016, 9906

[18] Srikanth S., Norrod R., King L., et al.: 'An Overview of the Green Bank Telescope', IEEE Antennas and Propagation Society International Symposium, Orlando, USA, Aug., 1999

[19]Feng S. F., Wang C. S., Duan B. Y., Ban Y.: 'Design of tipping structure for $110 \mathrm{~m}$ high-precision radio telescope', Acta Astronautica, 2017, 141, pp: 50-56

[20]Zhang J., Huang J., Zhou J., et al.: 'A compensator for large antennas based on pointing error estimation under a wind load', IEEE Trans. Antennas Propag., 2017, 25, (5), pp. 1912-1920

[21] Greve A., Bremer M.: 'Improvement of the IRAM 30-m telescope from temperature measurements and finiteelement calculations', IEEE Transactions on Antennas and Propagation, 2005, 53, (2), pp. 851-860

[22] Wang W., Wang C. S., Duan B. Y., et al.: 'Compensation for gravity deformation via subreflector motion of $65 \mathrm{~m}$ shaped cassegrain antenna', IET Microwaves, Antennas and Propagation, 2013, 8, (3), pp: 158-164 
[23] Nikolic B., Hills R. E., Richer J. S.: 'Measurement of antenna surfaces from in- and out-of-focus beam maps using astronomical sources', Astron Astrophys, 2007, 465, pp. 679-683

[24] Rahmat-Samii Y.: 'Surface diagnosis of large reflector antennas using microwave holographic metrology: An iterative approach', Radio Sci, 1984, 19, pp. 1205-1217

[25] Levy R.: 'Structural engineering of microwave antennas: for electrical, mechanical, and civil engineers' (NJ: IEEE Press, Piscataway, 1996)

[26] Zarghamee M.: 'Peak gain of a cassegrain antenna with secondary position adjustment', IEEE Trans. On Antennas and Propagation, 1982, 30, (6), pp: 1228-1233

[27] Rahmat-Samii Y.: 'An efficient computational method for characterizing the effects of random surface errors on the average power pattern of reflectors', IEEE Trans. Antennas Propag., 1983, 31, (1), pp. 92-98

[28]Ruze J.: 'Antenna tolerance theory - a review', Proceeding of IEEE, 1966, 54, (4), pp. 633-640 